\title{
Changing preventive practice: a controlled trial on the effects of outreach visits to organise prevention of cardiovascular disease
}

\author{
MEJL Hulscher, BB van Drenth, JC van der Wouden, HGA Mokkink, C van Weel, \\ RPTM Grol
}

Centre for Quality of Care Research, Universities of Nijmegen and Maastricht MEJL Hulscher, health scientist

BB van Drenth, general practitioner

HGA Mokkink, research methodologist

$C$ van Weel, professor of general practice RPTM Grol, professor of general practice

\section{Department of} General Practice, Erasmus University, Rotterdam

JC van der Wouden, sociologist

Correspondence to: Dr MEJL Hulscher, Centre for Quality of Care Research (WOK), University of Nijmegen, PO Box 9101 , $6500 \mathrm{HB}$ Nijmegen, The Netherlands.

Accepted for publication 16 January 1997

\begin{abstract}
Objectives-To assess the effects of outreach visits by trained nurse facilitators on the organisation of services used to prevent cardiovascular disease. To identify the characteristics of general practices that determined success.

Design-A non-randomised controlled trial of two methods of implementing guidelines to organise prevention of cardiovascular disease: an innovative outreach visit method compared with a feedback method. The results in both groups were compared with data from a control group.
\end{abstract}

Setting and subjects-95 general practices in two regions in The Netherlands.

Interventions-Trained nurse facilitators visited practices, focusing on solving problems in the organisation of prevention. They applied a four step model in each practice. The number of visits depended on the needs of the practice team. The feedback method consisted of the provision of a feedback report with advice specific to each practice and standardised instructions.

Main outcome measures-The proportion of practices adhering to 10 different guidelines. Guidelines were on the detection of patients at risk, their follow up, the registration of preventive activities, and teamwork within the practice.

Results-Outreach visits were more effective than feedback in implementing guidelines to organise prevention. Within the group with outreach visits, the increase in the number of practices adhering to the guidelines was significant for six out of 10 guidelines. Within the feedback group, a comparison of data before and after intervention showed no significant differences. Partnerships and practices with a computer changed more.

Conclusion-Outreach visits by trained nurse facilitators proved to be effective in implementing guidelines within general practices, probably because their help was practical and designed for the individual practice, guided by the wishes and capabilities of the practice team.

(Quality in Health Care 1997;6:19-24)

Keywords: guidelines; prevention of cardiovascular disease; general practice.
Introduction

Because of accessibility to a large segment of the population, general practitioners are in a favourable position to provide preventive services. Prevention of cardiovascular disease is among the priorities. ${ }^{1}$ Several studies showed that not only knowledge and skills are important for improving quality of care in prevention, but also the practical ability to detect and follow up patients at risk. They recommended implementing prevention by improving the organisation of preventive services..$^{2-7}$ We developed guidelines to organise prevention of cardiovascular disease, detection of patients at risk, their follow up, registration of preventive activities, and teamwork (table 1). The underlying assumption was that adherence to these guidelines would contribute to a decrease in cardiovascular morbidity and mortality.

Numerous reviews have focused on methods of implementing guidelines and improving quality of care. ${ }^{8-17}$ In general, they point to the low effectiveness of providing educational materials or traditional continuing medical education. Explicit guidelines, face to face education, feedback, or reminders seem to be effective, or at least promising, methods. Lomas and Haynes, ${ }^{9}$ Grol, ${ }^{12}$ and Wensing and $\mathrm{Grol}^{16}$ point to the particular effectiveness of intensive combined interventions.

One of the promising methods for improving the quality of care is face to face education or educational outreach visits by trained personnel providing individual instruction at the physician's surgery. It has been shown that this approach for optimising drug prescription and blood transfusion practice has been effective. ${ }^{10}{ }^{18-21}$ Outreach visitors were specialists who visited physicians, primarily to improve the physicians' knowledge. Similarly, monthly joint consultation sessions between general practitioners and an orthopaedic surgeon (for 18 months) resulted in more efficient care, with better targeted examination, treatment, and referrals. ${ }^{22} \mathrm{~A}$ related approach to prevention has been developed in the United Kingdom. It was reported that a nurse facilitator providing personal contact and focusing on specific organisational problems may increase (cardiovascular) preventive activity in primary care. $^{23-27}$ Other studies on prevention with nurse outreach visitors do not give a conclusive answer about the effectiveness of the model. ${ }^{28-31}$ 
Table 1 Guidelines for organisation of services to prevent cardiovascular disease

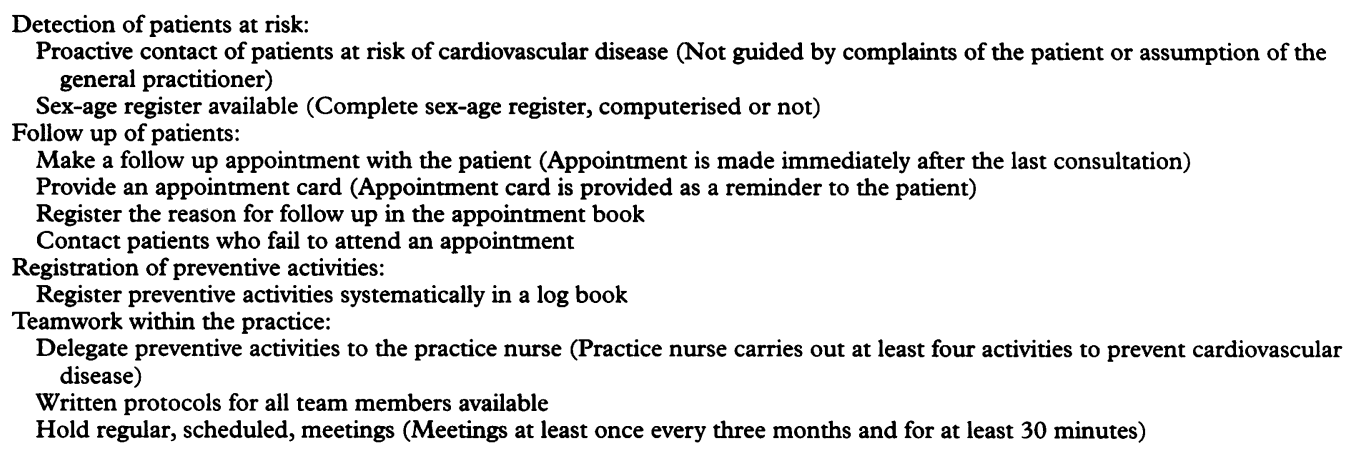

Table 2 Characteristics of the 95 participating practices by study group (\%)

\begin{tabular}{llll}
\hline Characteristics of practices & Outreach visits $(n=33)$ & Feedback $(n=31)$ & Control (n=31) \\
\hline Single handed & 39 & 42 & 45 \\
$\geq 2500$ patients/full time general practitioner & 45 & 42 & 45 \\
$\geq 0.8$ full time practice nurse/2500 patients & 73 & 77 & 81 \\
General practitioner involved in vocational training & 48 & 55 & 61 \\
Computer & 85 & 71 & 94 \\
Urban location & 55 & 52 & 61 \\
Age of the general practitioners in 1991 (mean(SD)) & $40.9(5.2)$ & $40.7(5.3)$ & $41.6(6.6)$ \\
\hline
\end{tabular}

In our study, we hypothesised that a trained nurse outreach visitor carrying out an intervention that combined various methods for quality improvement, would be successful in implementing prevention in general practice. The prevention of cardiovascular disease was treated as a case study. A model was developed in which a nurse facilitator was seen as the carrier of a set of interventions, focusing on implementing guidelines on prevention of cardiovascular disease. The aim of the study was to assess the effects of such outreach visits on the organisation of preventive strategies, and to identify characteristics of the practices that determined success. Because outreach visits are a rather novel method of implementation, the visits were compared with a feedback approach as used in many countries to improve quality of care. Through this comparison we could test our hypothesis that outreach visits would be more effective than the usual feedback approach.

This paper focuses on finding out the effects of outreach visits on adherence to guidelines to organise prevention of cardiovascular disease and what characteristics of the practices relate to those effects?

\section{Method}

GUIDELINES TO ORGANISE PREVENTION

Guidelines were derived from the Dutch College of General Practitioners' national guidelines on prevention of cardiovascular disease in general practice, and other relevant publications. $^{334}$ In a consensus procedure these guidelines were reviewed by general practitioners and practice nurses, to assess relevance, applicability, and feasibility. (Dutch practice nurses differ from, for example, British practice nurses. The tasks they carry out are administrative, or medical, or both.) Thus, a coherent set of guidelines was drawn up on detection of patients at risk (two guidelines), their follow up (four guidelines), registration of preventive activities (one guideline), and teamwork (three guidelines) (table 1).

\section{DESIGN AND PRACTICES}

Two methods of implementing guidelines to organise prevention of cardiovascular disease in general practice were compared: outreach visits by a trained nurse facilitator versus feedback. A comparison of both groups before and after the interventions was performed. The final assessments took place 18 months after the baseline assessment. To control for a time effect, a group without interventions was added. To avoid the possibility that baseline measurements would affect outcomes (Hawthorne effect), these control practices only had a final assessment.

A non-randomised controlled trial was carried out in 95 general practices in two regions in The Netherlands. No formal power analysis was performed. However, the minimal difference that can be assessed, given group sizes of 30 each and conventional choices for $\alpha$ $(0.05)$ and $\beta(0.20)$, is in the range of $35 \%-40 \%$ for unpaired comparisons. ${ }^{35}$ Practices were invited to participate in the study. Aiming at equivalent groups, the following criteria were used to assign practices to the three groups: type of practice, list size, employment rate of the practice nurse, and participation in vocational training. Table 2 shows data on characteristics of practices and providers. Data were collected from January 1991 until January 1994.

\section{STRATEGIES FOR IMPLEMENTATION}

Six practice nurses were selected based on their experience in general practice and on personal skills and were carefully trained to carry out the facilitator's role. They gained experience as facilitators in 12 pilot practices. In 33 practices, these six trained nurse facilitators carried out a combination of intervention 
Table 3 Outreach visits by facilitators (steps in the implementation process)

\begin{tabular}{ll}
\hline Steps & Content \\
\hline Step 1 Orientation & $\begin{array}{l}\text { Introductory visit involving all members of the practice team. Facilitator informs practice team on } \\
\text { prevention of cardiovascular disease and on guidelines for the organisation of prevention } \\
\text { Facilitator carries out a practice analysis to find out daily routines in relation to the guidelines and } \\
\text { writes a report }\end{array}$ \\
Discussion with practice team on the findings of the analysis, as reported in the feedback report \\
Step 3 Acceptance & $\begin{array}{l}\text { Facilitator and practice team draw up a plan of action, describing intended changes } \\
\text { Step 4 Change }\end{array}$ \\
& $\begin{array}{l}\text { Facilitator supports practice team in the implementation of planned changes } \\
\text { Facilitator provides tools to monitor progress } \\
\text { Facilitator gradually withdraws from the practice }\end{array}$ \\
\hline
\end{tabular}

methods, based on a theoretical model to diffuse and implement guidelines in four steps (table 3) and based on the principles of educational outreach. ${ }^{12} 32$ Firstly, the practice team was informed about the guidelines to gain their interest in the organisational aspects of prevention. Secondly, data were gathered on the organisation of prevention of cardiovascular disease. After this analysis of each practice, a personal feedback report was written by the nurse facilitator. The practice team and the nurse facilitator discussed the results of this analysis. The aim of this step was to give the team a better insight into their own situation and to create awareness of (gaps in) actual performance. Thirdly, the practice team, together with the facilitator, drew up a plan of action to optimise routines. Fourthly, the practice was supported to implement the planned changes during consecutive visits (box). If necessary, information or education was provided to improve knowledge, skills, or attitudes. Standardised instructions were provided if needed. Finally, visits were made to discuss progress and barriers so that changes could be consolidated. The practice was also provided with practical tools to self assess progress. During the last phase, which started some 12 months after the beginning of the intervention, the nurse facilitator gradually withdrew from the practice.

The facilitator applied this four step model in each practice. The number of visits, however, depended on the needs and wishes of the practice team. On average, visits involved about 30 hours of meetings for practice staff. Each practice was visited 25 times on average (SD 9) over a period of 18 months; the mean (SD) duration of these visits was 73 (41) minutes.

The feedback intervention (31 practices) consisted of analysis of the organisation of prevention of cardiovascular disease within each practice. After the same procedure as in the practices with outreach visits, data were gathered by questionnaire and one day of observation about the organisation of services used to prevent cardiovascular disease . Next, the practices were sent a written feedback report, providing information on the practices' daily routines and giving detailed advice on how to optimise the organisation. The report was sent to all members of the practice team, accompanied by standardised instructions.

\section{MEASUREMENTS}

Adherence to guidelines to organise prevention

Data on adherence to the guidelines to organise prevention (table 1) were gathered by ques-
Examples of activities that took place in a practice to improve organisation of services used to prevent cardiovascular disease

- Practice team reached agreement on protocols for measuring family history, blood pressure, cholesterol, weight, and smoking habits

- Practice nurse was trained to carry out consultations to prevent cardiovascular disease, that included both taking these measurements correctly and health counselling

- Specific tools-for example, questionnaires-were developed to take an accurate medical history

- Practice team selected and purchased leaflets for patients-for example, on diet and smoking

- Blood pressure devices were calibrated

- Appointment cards were developed and printed

- Suitable workroom was created for the practice nurse to see patients

- Practice nurse was trained to contact patients who fail to attend an appointment

- Logbook was developed to register preventive activities

- Practice team discussed progress and barriers in implementing guidelines for prevention

tionnaire and by observation (availability of sex-age register, reason for follow up in appointment book, log book, and written protocols), at baseline and after 18 months. Data on outcomes were dichotomised: a practice either did or did not adhere to the guideline. For each guideline the proportion of practices adhering was assessed.

\section{Characteristics of practices}

Information on characteristics of practices was collected by questionnaire at baseline. All practice variables were reduced to binomial variables:

- Type of practice (single handed $v$ any form of partnership)

- List size $(<2500 v \geq 2500$ patients per full time general practitioner)

- Employment rate of practice nurse $(<0.8 v$ $\geq 0.8$ per 2500 patients)

- Participation in vocational training (involved $v$ not involved) 
Table 4 Adherence to guidelines: percentages of practices in each study group adhering to the guidelines ( $n=95)$

\begin{tabular}{|c|c|c|c|c|c|}
\hline \multirow[b]{2}{*}{ Guideline } & \multicolumn{2}{|c|}{ Outreach visits $(n=33)$} & \multicolumn{2}{|c|}{ Feedback $(n=31)$} & \multirow[b]{2}{*}{ Control $(n=31)$} \\
\hline & Before & After & Before & After & \\
\hline \multicolumn{6}{|l|}{ Detection of patients at risk: } \\
\hline Proactive contact of patients at risk & 33 & $85^{\star \dagger}$ & 39 & $63^{\dagger}$ & 35 \\
\hline Sex-age register available & 64 & 76 & 65 & 77 & 84 \\
\hline \multicolumn{6}{|l|}{ Follow up patients: } \\
\hline Make a follow up appointment & 70 & $91^{\dagger}$ & 70 & 71 & 55 \\
\hline Provide an appointment card & 45 & $79^{\star \dagger}$ & 39 & 52 & 32 \\
\hline Register follow up consultations in appointment book & 31 & $72^{\star+}$ & 37 & $52^{\dagger}$ & 27 \\
\hline Contact patients who fail to attend an appointment & 15 & 27 & 10 & 16 & 16 \\
\hline \multicolumn{6}{|l|}{ Registration of preventive activities: } \\
\hline Use a log book & 0 & $73^{\star \dagger}$ & 3 & 7 & 3 \\
\hline \multicolumn{6}{|l|}{ Teamwork within the practice: } \\
\hline Delegate preventive activities to practice nurse & 18 & $73^{\star \dagger}$ & 19 & 35 & 19 \\
\hline Written protocols for practice team available & 6 & $94^{\star \dagger}$ & 3 & 10 & 6 \\
\hline Hold regular, scheduled, team meetings & 18 & 31 & 42 & 37 & 32 \\
\hline
\end{tabular}

${ }^{\star} \mathrm{P} \leq 0.05$ : before $v$ after intervention.

${ }^{\dagger} \mathrm{P} \leq$ 0.05: after $v$ control, Mann-Whitney $U$ test.

- Practice uses a computer (or not)

- Practice location (rural $v$ urban)

- Age of general practitioner(s) $(\leq 40 v>40)$ and practice nurse(s) $(\leq 30 v>30)$.

\section{ANALYSIS}

The unit of analysis was the practice. In analyses within groups, in which data were paired, the sign test was used. In analyses between groups, in which data were unpaired, Mann-Whitney $U$ tests were performed. The "difference of differences of proportions" between the two intervention groups was tested by $Z$ scores. ${ }^{36}$ To assess the influence of practice characteristics on the effects in both groups, before and after the assessment an unweighted sum score on adherence to the 10 specific guidelines was computed for each practice (score between 0 and 10). To correct the final sum scores for baseline measurements, residual values were derived by regressing the final sum scores on the baseline sum scores. Each characteristic of the practices was used as a grouping variable and was related to the residual final scores as a bivariate by unpaired $t$ tests, and by multiple stepwise regression analysis.

\section{Results}

CHARACTERISTICS OF STUDY GROUPS

The matching procedure was successful: the three groups of practices were similar for most of the matching variables. The control practices, however, used a computer more often (table 2). The three groups were also comparable at baseline for most of the guidelines: roughly the same percentages of practices adhered to the different guidelines (table 4). In the control practices an automatic sex-age register was more often available than in the other groups; the difference in computer use and in availability of a sex-age register can be ascribed to the increasing rate of computerisation among general practitioners (control practices were assessed after the test period). In the practices with outreach visits, baseline measures of team meetings were less favourable than in the feedback group $(18 \% v$ $42 \%, P=0.04)$.

\section{ADHERENCE TO THE GUIDELINES}

Final data on adherence to guidelines were gathered 18 months after the baseline data. In the group with outreach visits, the increase in the number of practices adhering to the guideline was significant $(P \leq 0.05)$ for six out of 10 guidelines (table 4). The increase in the number of practices adhering to the guideline to make a follow up appointment did not reach significance $(P=0.07)$. A comparison with the control group confirmed these outcomes, except that the difference for that guideline was now significant $(P<0.01)$.

Within the feedback group, a comparison between baseline and final data showed no significant increase in the percentage of practices adhering to any of the guidelines. Comparing final data with that of the control practices, differences were found for two guidelines: proactive invitations to patients at high risk of cardiovascular disease $(P=$ 0.03 ) and register follow up consultations in the appointment book $(P=0.05)$.

In the group with outreach visits, the increase in adherence to the different guidelines varied between $12 \%$ and $88 \%$ of the practices (table 5). In the practices with feedback, the changes varied between $-5 \%$ and $25 \%$. For all guidelines, the increase in the group with outreach visits was higher. These differences were significant $(P \leq 0.05)$ for four out of 10 guidelines.

EFFECTIVENESS AND PRACTICE CHARACTERISTICS To analyse the influence of several practice characteristics on the effectiveness of the implementation methods, the residual unweighted final sum score (corrected for unweighted baseline sum score (multiple $R=$ 0.24 ) was related to each practice characteristic as measured at baseline by $t$ tests (each practice characteristic being used as the grouping variable). The variables "type of practice" and "practice with a computer at baseline" were related to the residual final score; partnerships and practices with a computer at baseline complied more with guidelines. These two practice characteristics were not related $\left(\chi^{2}\right.$ 0.03, Pearson's $R-0.02$ ).

These outcomes were confirmed when practice characteristics were entered stepwise in a multiple regression analysis (table 6); partnerships and practices with a computer at baseline scored higher.

\section{Discussion}

Outreach visits were more effective than feedback in implementing guidelines to organ- 
Table 5 Change (4) in \% of practices adhering to the guidelines ( $n=64)$

\begin{tabular}{|c|c|c|}
\hline Guideline & Outreach visits $\Delta \%(n=33)$ & Feedback $\Delta \%(n=31)$ \\
\hline \multicolumn{3}{|l|}{ Detection of patients at risk: } \\
\hline Proactive contact of patients at risk & $52^{\star}$ & 25 \\
\hline Sex-age register available & 12 & 13 \\
\hline \multicolumn{3}{|l|}{ Follow up of patients: } \\
\hline Make a follow up appointment & 21 & 1 \\
\hline Provide an appointment card & 33 & 13 \\
\hline Register follow up consultations in appointment book & 41 & 15 \\
\hline Contact patients who fail to attend an appointment & 12 & 6 \\
\hline \multicolumn{3}{|l|}{ Registration of preventive activities: } \\
\hline Use a log book & $73^{\star}$ & 3 \\
\hline \multicolumn{3}{|l|}{ Teamwork within the practice } \\
\hline Delegate preventive activities to practice nurse & $55^{\star}$ & 16 \\
\hline Written protocols for practice team available & $88^{\star}$ & 6 \\
\hline Hold regular scheduled team meetings & 13 & -5 \\
\hline
\end{tabular}

$\star \mathrm{P} \leq 0.05$.

Table 6 Effectiveness of the two interventions and practice characteristics (multiple regression equation)

\begin{tabular}{llll}
\hline Variable & $B$ & $S E B$ & Significance T \\
\hline Computer at baseline (yes $=1$, no $=0$ ) & 2.01 & 0.66 & 0.004 \\
Type of practice (single handed = 1, any partnership =0) & -1.17 & 0.56 & 0.04 \\
Constant & -1.1 & 0.63 & 0.09 \\
\hline
\end{tabular}

Multiple $R=0.43 ;$ MR square $=0.18 ; F=6.9 ;$ Significance $F=0.002$.

ise services for the prevention of cardiovascular disease in general practice. The model of outreach visits by trained nurse facilitators, combining several effective methods, proved to be effective in improving the quality of care. Probably all elements of the intervention contributed to the effects: offering support designed for the individual practice, repeating messages during repeated visits, involving the practice team, feeding back behaviour, and offering the chance to try out several alternatives. Compared with this intervention, the feedback approach was much more static, although it included recommendations specific to the practice. The feedback method has often proved to be effective, and our results were disappointing. ${ }^{9-121416}$

In the practices that had outreach visits, seven guidelines were successfully implemented. They concerned the guidelines that are most closely linked to the actual performance of preventive activities: proactively contacting patients at risk, providing follow up appointments, registering preventive activities, and delegating tasks based on written protocols to the practice nurse. The implementation of two other guidelines proved to be difficult: contacting patients who failed to attend an appointment for cardiovascular disease and holding regular, scheduled, team meetings. We suppose that these two guidelines were harder to implement because they were less directly linked to the actual performance of preventive activities. Moreover, at baseline, adherence to the first guideline was low compared with the other follow up guidelines. Obviously, most practices hesitate to contact patients when they have missed an appointment, probably because of prevailing opinions among care providers on the patient's own responsibility. Regular, scheduled team meetings were also difficult to achieve. In most practices team meetings do take place, but on an ad hoc basis. Practice teams are probably not convinced of the extra value of meeting regularly on a scheduled basis. The increase in adherence to the guideline that a sex-age register is available must be due to the increasing rate of computerisation among general practitioners, and not to the intervention.

The influence of practice characteristics on the effectiveness of the implementation methods was analysed. Partnerships and practices with a computer showed more changes. Several explanations could be given for this. In partnership practices general practitioners can support each other to cope with barriers and to comply with the plan of action. Moreover, in a partnership practice there are more people to carry out the necessary activities. Another explanation could be that the computer itself made the implementation of organisational changes easier, because it provided facilities for certain procedures - for example, labelling patients at risk, providing the sex-age register, and labelling the patient's follow up appointment.

Practices were invited to participate in the study. Therefore, we may have selected motivated practices. This means that it may be harder to implement changes in unmotivated practices. This implies that simple, single moment methods - such as feedback - will almost certainly be insufficient to implement organisational changes.

Are outreach visits as effective an intervention for prevention of cardiovascular disease as they have been in other areas? We do not think that the effectiveness of the model depends on the subject considered. Maybe outreach visitors in the field of prevention should not, as for example in the study of Cockburn et $a l^{28}$ be merely seen as pharmaceutical representatives who want to sell a product in one or two visits. To successfully implement new ideas in general practice it is very important to support practices in applying a new approach, while going through all steps of the theoretical model to diffuse and implement this innovation (orientation, insight, acceptance, and change). ${ }^{12} \mathrm{~A}$ trained visitor can be effective by giving practical help designed for the individual practice, guided by the wishes and capabilities of the practice 
team. After the theoretical model of change, practices can be optimised within their own abilities and at their own pace. Given the huge adaptions that are often needed, repeated visits are necessary to offer several alternatives to be tried out.

In this study, we showed that outreach visits were effective in optimising the organisation of services in general practice. Prevention of cardiovascular disease was taken as a case study. To test our hypothesis that outreach visits are also effective in optimising patient care, a study was recently started in 120 general practices.

Facilitation is a rather expensive method of implementation. In this paper we reported that each practice was, on average, visited 25 (SD 9) times over a period of 18 months, with a mean total duration of about 30 hours. We only measured the hours a facilitator spent in a practice. To get a more realistic insight into the costs of the intervention - including the facilitator's travel expenses, hours of preparation, and the time devoted to the project by the practice team - the ongoing study also contains a cost analysis.

This study was supported by a grant from the Netherlands Heart Foundation (grant number: 13027 )

1 Fowler G. Coronary heart disease; prevention and risk factors. Practitioner 1989;233:423-6.

2 van den Hoogen JPH, van Ree JW. Preventive cardiology in general practice: computer-assisted hypertension care. $\mathscr{f}$ Hum Hypertens 1990;4:365-7.

3 Frame PS. Health maintenance in clinical practice: strategies and barriers. Am Fam Physician 1992;45:1192 200 .

4 Wender RC. Cancer screening and prevention in primary care. Cancer 1993;72:1093-9.

5 Costanza ME, D'Orsi CJ, Greene HL, Gaw VP, Karellas A Zapka JG. Feasibility of universal screening mammography. Lessons from a community intervention. Arch Inter Med 1991;151:1851-6.

6 Gann P, Melville SK, Luckmann R. Characteristics of primary care office systems as predictors of mammography utilization. Ann Intern Med 1993;118:893-8.

7 Elford RW, Jennett P, Bell N, Szafran O, Meadows L. Putting prevention into practice. Health Rep 1994;6:14253.

Haynes RB, Davis DA, McKibbon A, Tugwell P. A critical appraisal of the efficacy of continuing medical education. IAMA 1987;251:61-4

9 Lomas J, Haynes RB. A taxonomy and critical review of tested strategies for the application of clinical practice recommendations: from "official" to "individual" clinical policy. Am f Prev Med 1988;4(suppl):77-94

10 Soumerai SB, McLaughlin TJ, Avorn J. Quality assurance for drug prescribing. Quality Assurance in Health Care 1990;2:37-58.

11 Mugford M, Banfield P, O'Hanlon M. Effects of feedback of information on clinical practice; a review, BMf 1991; 303:398-402.

12 Grol R. Implementing guidelines in general practice care. Quality in Health Care 1992;1:184-91,
13 Davis DA, Thomson MA, Oxman AD, Haynes RB Evidence for the effectiveness of CME. A review of 50 randomized controlled trials. $\mathcal{F} A M A$ 1992;268:1111-7.

14 Buntinx F, Winkens R, Grol R, Knottnerus JA. Influencing diagnostic and preventive performance in ambulatory care by feedback and reminders. A review. Fam Pract 1993; 10:219-28.

15 Grimshaw JM, Russell IT. Effect of clinical guidelines on medical practice: a systematic review of rigorous evaluations. Lancet 1993;342:1317-22.

16 Wensing $M$, Grol R. Single and combined strategies for implementing changes in primary care: a literature review. Int f Qual Health Care 1994;6:115-32.

17 Grimshaw J, Freemantle N, Wallace S, Russell I, Hurwitz B Watt I, et al. Developing and implementing clinical practice guidelines. Quality in Health Care 1995;4:55-64.

18 Avorn J, Soumerai SB. Improving drug-therapy decisions through educational outreach. A randomised controlled trial of academically based "detailing". N Engl $f \mathrm{Med}$ 1983;308:1457-63.

19 Soumerai SB, Avorn J. Economic and policy analysis of university-based drug detailing. Med Care 1986;24:313 31

20 Soumerai SB, Avorn J. Predictors of physician prescribing oumerai SB, Avorn J. Predictors of physician prescribing change in an educational experiment

21 Soumerai SB, Salem Schatz S, Avorn J, Casteris CS, Ros Degnan D, Popovsky MA. A controlled trial of educational outreach to improve blood transfusion practice. $\mathscr{f} A M A$ 1993;270:961-6.

22 Vierhout WP, Knottnerus JA, van Ooij A, Crebolder HF Pop P, Wesselingh Megens AM, Beusmans GH. Effectiveness of joint consultation sessions of general practitioners and orthopaedic surgeons for locomotor-system disorders. Lancet 1995;346:990-4.

23 Fullard E, Fowler G. Promoting prevention in primary care controlled trial of low technology, low cost approach. $B M \mathcal{F}$ 1987;294:1080-2.

24 Fullard E, Fowler G, Gray M. Facilitating prevention in primary care, $B M \mathcal{F}$ 1984;289:1586-7.

25 Astrop P. Facilitator. The birth of a new profession. Health Visitor 1988;61:311-2.

26 Fowler G, Fullard E. Coronary heart disease: prevention and risk factors. Practitioner 1989;233:423-6.

27 Allsop J. Changing primary care: the role of facilitators. London: King's Fund Centre for Health Services Development, 1990

28 Cockburn J, Ruth D, Silagy C, Dobbin M, Reid Y, Scollo M Naccarella L. Randomised trial of three approaches for marketing smoking cessation programmes to Australian marketing smoking cessation programmes
general practitioners. $B M \mathcal{F}$ 1992;304:691-4.

29 Dietrich AJ, O'Connor GT, Keller A, Carney PA, Levy D, Whaley FS. Cancer: improving early detection and prevention. A community practice randomised trial. $B M f$ 1992;304:687-91.

30 Crotty M, Litt JCB, Ramsay AT, Jacobs S, Weller DP. Wil facilitators be acceptable in Australian general practice? A before and after feasibility study. Aus Fam Physician 1993; 22:1643-7.

31 Kottke TE, Solberg LI, Brekke ML, Conn SA, Maxwell P, Brekke MJ. A controlled trial to integrate smoking cessation advice into primary care: doctors helping smokers, round III. F Fam Pract 1992;34:701-8.

32 Soumerai SB, Avorn J. Principles of educational outreach (academic detailing) to improve clinical decision making. fAMA 1990;263:549-56.

33 Thomas S. Standard setting in the Netherlands: impact of the human factor on guideline development. $\mathrm{Br} \mathcal{f} \mathrm{Gen}$ Pract 1994;44:242-3.

34 Grol R, Thomas S, Roberts R. Development and implementation of guidelines for family practice: lessons from the Netherlands. F Fam Pract 1995;40:435-9.

35 Fleiss JL. Statistical methods for rates and proportions. New York: Wiley, 1981.

36 Blalock HM Jr. Social statistics. Tokyo: McGraw-Hill Kogakusha, 1972. 